\title{
Littératies numériques : quels enjeux pour la didactique de l'écriture-lecture?
}

Réponse à Jeannine Gerbault

\section{Marie-Claude Penloup}

\section{(2) OpenEdition}

Journals

Édition électronique

URL : http://journals.openedition.org/rdlc/3900

DOI : $10.4000 /$ rdlc.3900

ISSN : 1958-5772

Éditeur

ACEDLE

Référence électronique

Marie-Claude Penloup, « Littératies numériques : quels enjeux pour la didactique de l'écriturelecture?», Recherches en didactique des langues et des cultures [En ligne], 9-2 | 2012, mis en ligne le 10 septembre 2018, consulté le 19 avril 2019. URL : http://journals.openedition.org/rdlc/3900 ; DOI $10.4000 /$ rdlc.3900

Ce document a été généré automatiquement le 19 avril 2019

\section{(†)

Recherches en didactique des langues et des cultures is licensed under a Creative Commons AttributionNonCommercial-NoDerivatives 4.0 International License 


\title{
Littératies numériques : quels enjeux pour la didactique de l'écriture-lecture?
}

\author{
Réponse à Jeannine Gerbault
}

Marie-Claude Penloup

\section{Introduction}

1 Intervenante au titre de réactante dans cette journée sur les Littératies, je tiens d'abord à remercier Danièle Moore et Muriel Molinié pour leur invitation ainsi que Jeannine Gerbault pour sa communication stimulante qui brasse nombre d'informations et nombre de questions vives sur ces nouvelles littératies qui émergent de l'internet et des autres techniques d'information et de communication. Ma réaction portera moins sur une discussion des constats qui nous sont apportés et de l'état de l'art qui nous est proposé que sur un prolongement de ce qui est abordé en troisième partie, tout en resserrant le propos dans une perspective très étroite au regard de l'ampleur des problèmes posés. Ma réflexion s'inscrit en effet dans le seul champ de la didactique du français écrit et porte sur la manière dont cette dernière est interpellée par l'exigence de l'acquisition d'une compétence numérique par les apprenants.

2 La notion de littératie s'invite dans le champ de la didactique du français depuis une quinzaine d'années, la réflexion portant en particulier sur ce qu'apporte la notion dans ce domaine de recherche (Barré-de Miniac, Brissaud, Rispail, 2004) et sur ses effets sur la formalisation de l'écrit (Reuter, 2006). Indiscutablement, la notion incite à une " reconfiguration des problèmes d'apprentissage et d'enseignement de la lecture/écriture dans une didactique de la culture écrite » (Chiss, $2011: 10$ ). La question des littératies numériques nous donne l'occasion de poser quelques-uns de ces problèmes. On notera ici que j'ai substitué le pluriel au singulier. De fait, si le choix du singulier traduit une opposition d'ordre technologique tout à fait évidente entre littératie électronique et littératie traditionnelle, il ne permet pas de rendre compte d'autres lignes de clivage : entre les 
pratiques dominantes de la littératie, soutenues par l'école (Barton, Hamilton : 50) et les littératies vernaculaires d'une part, entre les genres d'écrits d'autre part: il y a par exemple peut-être davantage de liens entre un blog électronique et un journal intime papier qu'entre un blog et un chat.

3 À partir des « questions ouvertes et sensibles » que pointe J. Gerbault et que je reprendrai partiellement pour les déplier, je centrerai mon propos sur les nouveaux usages de l'écrit que l'enseignant de français a à prendre en compte.

Deux questions organisent ma réflexion :

- qu'est-ce qui, dans les nouveaux usages de l'écrit qu'induit la révolution numérique, peut et doit faire l'objet d'un enseignement-apprentissage à l'école dans le cadre du cours de français?

- qu'est-ce que les outils numériques apportent à l'enseignement du français ?

Les réponses apportées à ces deux questions m'amèneront à envisager, dans un troisième temps, les modifications dans les gestes professionnels et surtout dans la posture de l'enseignant de français qu'induisent les littératies numériques.

\section{Les littératies numériques, objets d'apprentissage dans la classe de français?}

6 Comme le soulignent les différents rapports que cite J. Gerbault dans sa communication, l'apprenant du nouveau millénaire, plongé dans un monde dans lequel les environnements numériques ne cessent de se développer, a besoin de maitriser le nouveau monde des médias numériques. Cette compétence revêt des enjeux sociaux (le monde change, il faut répondre à une compétition économique globale dans un âge de l'information: Leu, 2002) et scolaires, ne serait-ce que parce qu'il y a désormais des certifications imposées dans ce domaine ${ }^{1}$.

7 Or, comme le notait R. Chartier dans sa leçon inaugurale au Collège de France, la « révolution numérique » "en brisant le lien ancien noué entre les discours et leur matérialité [...] oblige à une radicale révision des gestes et des notions que nous associons à l'écrit » (cité par Benamou, 2009: 82).

\section{De nouveaux gestes face à l'écrit}

F. Benamou (ibid.), reprenant les propos de R. Chartier, évoque les «deux bouleversements » que connaît la lecture, l'un étant qu'elle subit « un processus de fragmentation », l'autre qu'elle acquiert une dimension interactive. A quoi il faut rajouter ce que relève J. Gerbault, à savoir le fait que le caractère multimodal de la communication électronique nécessite le développement de compétences spécifiques.

9 L'écriture est concernée, comme la lecture, par l'interactivité propre à la communication électronique. Elle est, par ailleurs, affectée dans sa forme même dans la mesure où, à côté du français standard, se développe un français dit «électronique » ou encore «langage SMS » caractérisé par un certain nombre de procédés scripturaux liés à la fois à des besoins techniques (jusqu'il y a peu les messages sur les téléphones portables ne devaient pas comporter plus de soixante signes) et à des manifestations identitaires. Trois processus peuvent ainsi être observés ${ }^{2}$ : 

écrite et la culture par le fait qu'elle risque d'engendrer la marginalisation des pratiques normées et donc, un appauvrissement de l'écriture qui peu à peu « souffrirait de n'être plus ou seulement peu pratiquée dans sa forme la plus riche » (Jalabert, 2006). causes, au développement de l'écriture électronique? Ces questions méritent d'être posées et approfondies, loin de toute prise de position idéologique. Elles ne dispensent pas pour autant, et ce quelles que soient les réponses apportées, d'une réflexion sur le 
statut à donner, dans la classe de français, à cette variété de l'écrit qu'est le français électronique. Mon point de vue, alimenté par toute la réflexion de la sociolinguistique scolaire et de la sociodidactique est qu'il en est de cette variété comme d'autres: il appartient à l'école de la situer, en contexte, comme un usage possible de l'écrit qui s'écarte, certes, du français standard normé exigé à l'école mais qui a sa pertinence et peut servir de support à une prise de conscience des questions de norme et de variations, hors de toute stigmatisation des usagers.

19 Les données issues des littératies numériques aident à fonder scientifiquement et matériellement une critique toujours à faire de la représentation uniciste et donc réductrice de la langue française, et tout particulièrement de la langue de l'écrit, dominante en milieu scolaire malgré une réflexion en didactique dans ce domaine déjà ancienne. Les écrits numériques permettent, à double titre, une ouverture sur la «plurilittératie »: en ce qu'ils développent une variété de l'écrit différente de la variété standard mais aussi parce que l'une de leurs caractéristiques est manifestement de mettre en contact de manière très fréquente les langues qui font partie du répertoire d'un usager.

Et n'est-ce pas encore de plurilittératie que fait montre mon fils aîné, s'interroge M. Rispail, quand il m'écrit sur son téléphone portable : thanks a lot, va molto bene, bn kour alora [...] ( = merci beaucoup, ça va très bien, bon cours alors [...]) ? (Rispail, 2011 : 8).

20 Comme le note I. Pierozak à propos de ce qu'elle nomme les « espaces communautaires électroniques » : ils " permettent aux enfants uniquement francophones d'expériencer une compétence plurilingue et pluriculturelle, notamment au plan graphique » (Pierozak, 2010 : 93). Le rôle du professeur de français est triple : il s'agit de faire prendre conscience de la variation, de la faire analyser aux plans linguistique et pragmatique et d'y entraîner de manière réflexive (exercices de transcodage d'un SMS en français standard par exemple).

\section{Les TIC : outils pour la classe de français ${ }^{3}$}

Simply having internet technologies in our classrooms will not prepare children adequately for the new litteracies they require [...] leadership in preparing teachers to use these technologies for instruction is often lacking in nations around the world (Leu, 2000).

21 Même si, comme le note D. Leu, le défi d'une formation des enseignants à l'utilisation des nouvelles technologies de l'information et de la communication n'est pas encore relevé au niveau où on pourrait l'espérer, de nombreux travaux montrent, pour la didactique du français, combien ces dernières sont susceptibles de modifier le paysage de l'enseignement-apprentissage du français écrit. Je relèverai en particulier quatre domaines de transformation :

- Au plan cognitif, comme le souligne J. Gerbault, l'existence de l'hypertexte modifie les formes d'apprentissage de la littérature mais le traitement de texte, à lui seul, modifie la production écrite. Et ce à deux niveaux : d'une part, le traitement de texte joue un rôle facilitateur dans l'enseignement-apprentissage des pratiques de révision et de réécriture (Audran, Del Perugia, 2000) grâce à ses fonctions d'édition (remplacer, supprimer, déplacer) qui familiarisent avec la transformation du texte (ajout, déplacement, effacement); d'autre part, il libère le scripteur de certains aspects de la production : de 
certains actes moteurs propres à l'écriture manuscrite (Ameka, 2006:64) et du travail de correction orthographique le temps de l'écriture, grâce à l'existence de correcteurs.

- L'usage des TIC est propre, par ailleurs, à diminuer l'insécurité scripturale des apprenants. On sait, depuis les travaux de M. Dabène (1987) que le "passage à l'écriture ", auquel nous avons consacré un ouvrage (Delamotte, Gippet, Jorro, Penloup, 2000), met tout usager de l'écriture en situation anxiogène, de manière constitutive, car il est nécessairement pris dans un réseau de tensions entre deux pôles. Un pôle positif: attraction, communication, protection du territoire. Un pôle négatif : exposition de soi, évaluation etc. L'évaluation des écrits et les sanctions des écarts par rapport à la norme ont pour effet de majorer l'anxiété et, dans le pire des cas, d'inciter l'usager à éviter la prise de risque que signifie le passage à l'écriture.

Provoquer, à l'école, une communication électronique dont les usagers savent de plus en plus qu'elle accepte des normes plus souples mais aussi que le professeur ne les maitrise pas mieux que les élèves (souvent moins bien), c'est donner sa chance de passer par-delà le blocage lié à une maîtrise de la langue incertaine pour aller vers la communication écrite. Je renvoie sur ce point à l'expérience développée par F. Liénard (2007) en formation avec des adultes dits en situation d'illettrisme.

- Par ailleurs, et c'est en lien d'ailleurs avec le point précédent, les TIC amènent à mettre en place des pratiques de communication «authentiques» (Chenevez, 2001), plus motivantes. Ces dernières incitent l'élève à tenir compte des conditions de réception et en particulier, des destinataires, ce qui peut éviter nombre de malentendus comme c'est le cas avec l'exercice traditionnel de la rédaction déconnecté de toute situation de communication, et contribuer à donner sens au soin apporté à la production d'écrit, y compris en matière d'orthographe :

Les élèves sont conduits à écrire davantage pour les autres, ce qui les oblige à viser une plus grande clarté de l'expression, une meilleure mise en page et (en principe !

), une meilleure orthographe. (Ameka, $2006: 64-65$ ).

26 - Dans le même temps, enfin, se mettent en place des pratiques collaboratives, qui, tout en ayant un effet de sécurisation, permettent l'acquisition de savoirs d'ordre métalinguistique et métacognitif, et favorisent le développement de compétences langagières et rédactionnelles : les élèves acquièrent par un oral de coopération et de «friction 》 (Marty, 2005) des connaissances sur l'acte de parler, de convaincre, de négocier par exemple (Audran, Del Perugia, $2000: 8$ et 10). Communication et collaboration sont les deux avantages pointés par J. Crinon et D. Legros (2000: 163-164), ces derniers faisant référence aux travaux de Jones et Pellegrini (1996), auprès d'élèves de première année de l'école élémentaire, pour attester "des effets des relations sociales et du média sur les processus métacognitifs » lors d'une tâche d'écriture; effets marqués par un nombre plus important de conversations métacognitives "caractérisées par l'emploi plus fréquent d'expressions comme 'I think, I know, I guess' ou des articulateurs logiques, 'although, because' " (Crinon, Legros, $2000: 166-167)$. N. Marty (2005: 110) aussi a recueilli plusieurs types de données métalinguistiques: conduites de «survol » linguistique amenant à corriger intuitivement, remarques d'ordre métalinguistique de type « grammatical » et remarques d'ordre métalinguistique qui accompagnent les conduites de reformulation. La didactique de l'écrit semble donc avoir à gagner à utiliser ces " merveilleuses machines à communiquer " (Marty, 2005: 152) afin d'aller vers des pratiques d'écriture plus collectives et communicatives, vers des écrits qui intègrent «l'importance de la dynamique communicative et interactive» (53). 
27 Cela suppose, on l'a évoqué, chez les enseignants l'acquisition de gestes professionnels spécifiques qu'évoque pour partie J. Gerbault (accompagner un étudiant sur un forum de discussion, repenser les enseignements à distance, utiliser des plate-formes comme moodle etc.) mais c'est surtout sur la modification de posture que cela suppose que je voudrais insister pour finir.

\section{L'incidence des littératies numériques sur les postures des enseignants face aux apprenants}

Introduite par D. Bucheton, la notion de " posture " renvoie à celle de « schèmes d'actions cognitives et langagières disponibles, préformées, que le sujet convoque en réponse à une situation rencontrée » (Bucheton, Chabanne, $1998: 20$ ). Elle convient bien, dans le cadre de réflexion qui est ici le mien, pour évoquer la manière dont les littératies numériques sont susceptibles d'affecter en profondeur non seulement les représentations qu'ont les enseignants de leurs élèves et des modes d'apprentissage de l'écrit mais aussi les gestes professionnels qu'ils ont incorporés.

J'ai ainsi évoqué la manière dont l'existence des littératies numériques et le fait que leur acquisition se fasse largement hors école interdisent de penser l'écrit comme le domaine réservé de cette dernière mais contraignent, au contraire, à penser le rôle spécifique de l'enseignant de français dans ce cadre. Dit autrement, cela signifie que la prise en compte de l'apprenant ne se fait pas sur le seul mode de l'objectivation de ses lacunes ou des représentations qui jouent le rôle d'obstacles mais qu'elle conduit aussi à la mise au jour de ses connaissances. Avec les littératies numériques, il devient impossible d'ignorer la réalité massive et indiscutable d'une écriture enfantine et adolescente en dehors de l'espace scolaire et, surtout, de faire abstraction des connaissances langagières et techniques qu'elle suppose et qui sont, sur certains points, plus assurées chez l'apprenant que chez l'enseignant. Ce dernier se retrouve alors dans l'impossibilité de se cantonner au seul schéma descendant d'un enseignement délivré par un maître, doté de savoir, à un apprenant ignorant. L'expertise du maître n'est pas remise en cause mais elle peut tenir moins au savoir qu'il apporte qu'à celui qu'il reconnaît chez l'apprenant et dont il l'aide à prendre conscience, à le faire passer de la " connaissance » intériorisée et implicite à un "savoir " qui résulte, lui, d'un processus d'objectivation de la connaissance et de sa théorisation dans un langage approprié, explicite et, dès lors transférable.

De façon encore plus évidente sans doute que les écrits papiers, les écrits électroniques extrascolaires d'enfants et d'adolescents se prêtent à la reconnaissance par les enseignants de ce que nous nommons des " connaissances ignorées", au double sens de « insues » et de «méprisées » et induisent, de ce fait, une forme de retournement dans la posture des enseignants et dans les frontières qui s'établissent entre eux et les apprenants :

Ce qui précède contribue à abolir en partie la frontière entre apprenants et enseignants (puisque nous sommes TOUS en marche) mais aussi parce que l'interaction entre ces deux catégories d'acteurs, présentées souvent comme étanches, participe à la dynamique de la littéracie.[...] Littéraciquement parlant [elle évoque les textos], on apprend tous les uns des autres et le champ apparait comme un construit social : où le clivage entre théorie et pratique perd sa raison d'être. Si on se place dans le cadre scolaire, le point de vue littéracique pourrait renouveler notre vision de l'élève et de ses capacités, voire nos objectifs d'enseignement (Rispail, $2011: 3$ ). 


\section{Conclusion}

31 A la question posée par J. Gerbault de savoir si la littératie numérique peut aider à construire la littératie traditionnelle, on voit que je réponds positivement, d'une part parce que les outils numériques offrent des moyens d'accentuer l'exposition à l'écrit, de motiver et de sécuriser les usagers de l'écrit et, d'autre part, parce qu'ils invitent à des modifications dans les modes d'enseignement-apprentissage et dans la posture adoptée par les enseignants. Ces derniers étant amenés, en particulier, à développer des formes de travail plus collaboratives et une expertise pour nommer les connaissances des apprenants.

Les questions que posent les littératies numériques ne sont pas, au fond, totalement inédites dans le domaine de l'enseignement-apprentissage du français écrit mais elles sont plus difficiles à ignorer parce que plus urgentes et plus visibles. Elles sont, en ce sens, une chance pour la didactique du français.

\section{BIBLIOGRAPHIE}

Ameka, B. et al. (2006). Dictionnaire des nouvelles technologies en éducation. Paris : Nathan.

Anis, J. (dir). (2001). Parlez-vous texto ? Paris : Le Cherche-Midi.

Audran, J., Del Perugia, B. (14-18 novembre 2000). « Les chats à l'école : quels apprentissages ?», Colloque CIME-ICEM (International Council for Educational Media) et Media Days, Éducation et médias, le virage du numérique. Université de Genève. http://www.audran.net/articles/ CIME2000.PDF

Barré-de Miniac, C., Brissaud, C., Rispail, M. (2004). La littéracie, Conceptions théoriques et pratiques d'enseignement de la lecture-écriture. Paris : L'Harmattan.

Barton, D., Hamilton, M. (2010). «La littératie : une pratique sociale ». Langage et société, $\mathrm{n}^{\circ} 133$, pp. 45-62.

Benamou, F. (mars-avril 2009). « Le livre numérique. Ni tout à fait le même, ni tout à fait un autre... ». Esprit, $\mathrm{n}^{\circ} 3-4$, pp. 73-85.

Bucheton, D., Chabanne, J-.C. (1998). « Le point de vue, le doute et le savoir ». Le Français Aujourd'hui, $\mathrm{n}^{\circ} 123$, pp. 16-28.

Chenevez, O. (septembre 2001). «Ah ! si Freinet avait connu le web... ». Les Cahiers Pédagogiques, n -396, pp. 49-50

Chiss, J-L. (2011). « Littératie et didactique de la culture écrite » , forumlecture.ch n 1, pp. 1-12 (1 ère publication dans Pratiques 137/138, pp. 165-178). www.forumlecture.ch

Crinon, J, Legros, D. (2000). « De l'ordinateur outil d'écriture à l'écriture outil » . Repères, $\mathrm{n}^{\circ} 22$, pp. 161-173. 
Dabène, M. (1987). L'adulte et l'écriture. - Contribution à une didactique de l'écrit en langue maternelle. Bruxelles : De Boeck-Wesmael.

Delamotte, R., Gippet, F., Jorro, A., Penloup, M-C. (2000). Passages à l'écriture. Paris : PUF.

Dinet, J., Tricot, A. (2008). « Recherche d'information dans les documents électroniques» . In Chevalier, A., Tricot, A. (dir). Ergonomie des documents électroniques. Paris : PUF, pp. 35-69.

Jalabert R. (février 2006). »MoliR revi1 vit ... il son 2venu foo !« . Les Cahiers pédagogiques, $\mathrm{n}^{\circ} 440$. http://www.cahiers-pedagogiques.com/article.php3?id_article=2165

Joannidès, R. (2009). Et si on tchatait après la récré ? Réflexion sur les enjeux et modalités du tchat à l'école. Mémoire de Master 2 en Sciences du langage, Université de Rouen.

Leu, D.J. Jr. (2000) « Our children's future : changing the focus of literacy and literacy instruction ». The Reading Teacher, 53, pp. 424-431

Liénard, F. (2007). »Au-delà des faiblesses en langue, l'entrée dans une pratique communicationnelle d'adultes en formation « In Penloup, M-C. (dir.). Les connaissances ignorées Approche pluridisciplinaire de ce que savent les élèves. Lyon : INRP, pp. 129-150.

Liénard., F., Penloup, M-C. (2008). « Le rapport à l'écriture, un outil pour penser la place de l'écriture électronique dans l'enseignement-apprentissage du français » In Alamargot, D., Bouchand, J. Lambert, E., Millogo, V. et Beaudet, C. (dir). Proceedings of the international conférence De la France au Quebec : l'écriture dans tous ses états. Poitiers : France, 12-15 nov. 2008. http:// www.ecritfrancequebec2008.org/

Marty. N. (2005). Informatique et nouvelles pratiques d'écriture. Paris : Nathan.

Penloup, M-C. (dir). (2007). Les connaissances ignorées - Approche pluridisciplinaire de ce que savent les élèves. Lyon : INRP.

Pierozak, I. (2010). « Espaces communautaires électroniques et plurilinguisme : quels enjeux didactiques? ». Les Cahiers de l'Acedle, volume 7, n 1, pp. 83-96.

Rispail, M. (2011). "Littéracie : une notion entre didactique et sociolinguistique -enjeux sociaux et didactiques ». forumlecture.ch $n^{\circ} 1 / 2011$, pp. 1-11. www.forumlecture.ch

\section{NOTES}

1. Exemples pour la France : B2i, C2i.

2. Voir sur ce point : Lienard, Penloup, 2008.

3. Cette partie a été écrite à partir du mémoire de R. Joannidès (2009).

\section{RÉSUMÉS}

La révolution numérique a introduit des écrits d'un nouveau genre ainsi que de nouveaux gestes liés à l'écrit qu'il s'agit pour l'apprenant de maîtriser. Elle fournit, dans le même temps, de nouveaux outils pour l'enseignement-apprentissage de l'écriture-lecture. C'est dans ce double cadre que sont envisagées les questions posées à la didactique du français écrit par les littératies 
numériques. L'accent est mis sur le changement de posture qu'impliquent, chez l'enseignant, ces nouvelles formes de littératie et sur le gain que peut alors constituer leur irruption dans le cadre scolaire pour l'ensemble de la réflexion sur la didactique du français écrit, qu'il soit ou non électronique.

The digital revolution has introduced new kinds of written genres as well as new forms related to writing that the learner has to master. It provides, at the same time, new tools for the teaching and learning of reading and writing. It is within this double framework that the questions of teaching written French using digital literacies are evaluated. The emphasis is on the changing attitudes that these new forms of literacy imply for teachers and the gains that could be attained in the school context from a global reconsideration of the teaching of French writing, whether it be electronic or not.

\section{INDEX}

Keywords : digital literacy, electronic writing, learning, ICT, hidden knowledge

Mots-clés : littératie numérique, écriture électronique, didactique, TIC, connaissances ignorées.

\section{AUTEUR}

\section{MARIE-CLAUDE PENLOUP}

Université de Rouen, Laboratoire LiDiFra, France.

Marie-Claude Penloup est professeure en sciences du langage et de la communication à l'université de Rouen. Elle est membre du laboratoire LIDIFRA (Linguistique et Didactique du Français) et participe aux comités de rédaction ou de lecture des revues suivantes : Repères, Recherches en Didactique, Le Français Aujourd'hui, Lidil.

L'essentiel de son enseignement et de ses travaux de recherche porte sur la description des pratiques d'écriture spontanées des enfants et adolescents, hors contexte scolaire, et sur la manière dont l'objectivation de ces pratiques vient interroger la didactique de l'écriture, en particulier sous l'angle du rapport à l'écriture des apprenants et des enseignants.

Courriel : Marie-Claude.Penloup[at]univ-rouen.fr

Adresse : UFR de Lettres et Sciences Humaines, Rue Lavoisier, 76821 Mont Saint Aignan, France. 